\title{
Crucial inputs to nucleosynthesis calculations
}

\author{
T Rauscher \\ Dept. of Physics and Astronomy, University of Basel, Basel, Switzerland \\ E-mail: Thomas.Rauscher@unibas.ch
}

\begin{abstract}
The first part of the paper discusses nuclear properties relevant to predict compound reactions. The second part addresses direct reactions with special emphasis on direct neutron capture.
\end{abstract}

\section{Introduction}

Reaction rates are central in the modeling of nucleosynthesis and astrophysical energy generation and are computed by an integration of reaction cross sections across an energy range defined by the plasma temperature. Due to the comparatively low energies ( $E \lesssim 10 \mathrm{MeV}$ for charged projectiles and $E \lesssim 1 \mathrm{MeV}$ for neutrons) relevant in astrophysical plasmas, mainly three reaction mechanisms are important [1]: compound, resonant, and direct reactions. In principle, both resonant and direct mechanism may contribute to the reaction cross section although one of them is negligible in most cases. The nuclear level density in the compound nucleus, at the energy at which the compound nucleus would be formed, determines which mechanism is dominating [2]. The statistical Hauser-Feshbach model can be applied when there are many, overlapping resonances which can be treated with averaged transmission coefficients. In the absence of resonances and strong tails of resonances, the direct mechanism will be important even at low energy [1]. In between the two regimes, the contributions of single resonances require the precise knowledge of their properties, including energy, spin, parity, as well as the phases of wave functions to account for possible interference effects.

Describing the interplay of different mechanisms has been a longstanding problem in the study of nuclear reactions and requires precise predictions of excited state properties. A thorough discussion is beyond the scope of this paper but it is emphasized that this, among other things, shows the great importance of measuring cross sections in the relevant energy range. Here, we are focusing on some of the ingredients necessary for calculations in the Hauser-Feshbach model. One of the main difficulties in the determination of reaction rates for astrophysics lies in the fact that most reactions involve unstable nuclei which are not (yet) accessible in the laboratory. Therefore, the required properties cannot be extracted from experiment but rather have to be predicted by theory. In the second part of the paper we discuss direct capture and compare the results to Hauser-Feshbach cross sections for selected nuclides. 


\section{Compound reactions}

\subsection{Important nuclear properties}

Averaged widths (or transmission functions) are the central quantities in the calculation of cross sections in the Hauser-Feshbach model [3]. The relevance of the nuclear level density not only for the identification of the reaction mechanism but also for the computation of transmission functions has been discussed elsewhere [1, 2, 4]. The sensitivity of the results to variations in the level density is not strong because $i$ ) transitions to low lying states are dominating and these are usually explicitly included [5, 6], and $i i$ ) the thermal population of target states in the plasma allows for additional transitions washing out the effect of non-equally distributed parities [4].

Averaged radiative widths are usually calculated including E1 and M1 transitions [7, 5, 8], more recently also E2 9]. For E1 transitions, Lorentzian shapes with modified low-energy tails [5] or strength functions from QRPA calculations [10] are used. Frequently, the appearance of low-lying additional GDR strength is discussed, e.g. stemming from soft-mode vibrations, often termed "pygmy resonance". This could, in principle, strongly enhance neutron capture cross sections of very neutronrich nuclei [8, 10]. However, it will only be important for r-process nucleosynthesis if three conditions are met: $i$ ) the additional strength has to be located below the neutron separation energy of the nucleus because otherwise there will be no $\gamma$ transitions affected, $i$ i) the statistical model has to be applicable because otherwise direct capture may be dominating which does not see the GDR+pygmy strength, and iii) the involved nuclei should not participate in reaction equilibria $((\mathrm{n}, \gamma)-(\gamma, \mathrm{n})$ equilibrium $)$ but rather be produced by individual captures or photodisintegrations. The last item calls for nuclei located outside the main r-process path which are reached in the brief freezeout phase. There is no consistent picture yet. Previous microscopic calculations found some strength below the neutron threshold [11], while recent calculations find the pygmy resonance consistently several $\mathrm{MeV}$ above the neutron separation energy [12, 13, 14, 15, 16. The latter would rule out an effect on astrophysical rates provided the width is not so large as to reach below the separation energy. The location of the additional strength above the separation energy for Sn isotopes is confirmed by a recent experiment [17, 18, 19]. Earlier experiments found additional E1 strength below the separation energy in semi-magic isotopes of $\mathrm{Ba}, \mathrm{Ce}$, and $\mathrm{Sm}$ [20]. Future experiments have to test the predictions further away from stability.

Another complication in the prediction of $\gamma$ transitions arises due to isospin selection rules suppressing E1 and M1 $T=0 \rightarrow T=0$ transitions. This leads to a strong suppression of $(\alpha, \gamma)$ cross sections of self-conjugate nuclei [21] and some suppression of $(\mathrm{p}, \gamma)$ and $(\mathrm{n}, \gamma)$ on such nuclei. Often, this effect is included by introducing arbitrary suppression factors [8, 22] because the original Hauser-Feshbach equation implicitly assumes complete isospin mixing [3]. It is possible to generalize the model to explicitly treat isospin mixing [7, 21].

Nuclear masses determine the reaction $Q$-values in the open channels. Beyond the 
region of measured masses, theoretical models have to be employed. Special attention has to be paid to the transition from the measured to the unmeasured region when computing $Q$-values. Special care has to be taken to avoid artificial breaks in the relative channel energies when subtracting values from different sources.

\subsection{Optical potentials}

Transmission functions for particles are usually predicted by utilizing the optical model [1]. This requires an optical potential with real and imaginary parts that are, in principle, dependent on the type of the projectile, target mass, and projectile energy. Because of the sensitivity of the transmission coefficients to the depth and geometry of a potential, the calculated cross sections are also very sensitive to the choice of potential. Global potentials are necessary for large-scale predictions which should also give reliable results off stability where no experimental information is available.

The largest uncertainties in this respect are in optical potentials for charged projectiles due to the low incident energies occurring in astrophysical applications. The relevant energies are close to the Coulomb barrier. This makes it extremely difficult not just to measure cross sections but also to determine optical potentials by scattering [23. Available global potentials are usually derived at higher energies and/or only for a limited mass range.

The microscopic optical potential of [24] (JLM) with low-energy modifications by [25] is widely used for neutrons and protons. With a few exceptions it has been successful in reproducing experimental reaction data across a large range of target masses. Recently, new parameterizations of the JLM potential have been derived [26, 27] with the aim to improve its performance at energies above $160 \mathrm{MeV}$. However, this new potential does not work at low energies, as has been found in several comparisons to recent data [28, 29], whereas the JLM potential performed much better. However, it has been argued that the isovector components of the JLM potential may be too weak [26]. Indeed, in a comparison to recent ${ }^{70} \mathrm{Ge}(\mathrm{p}, \gamma)$ and ${ }^{76} \mathrm{Ge}(\mathrm{p}, \mathrm{n})$ data [29] we find that the absolute value and the energy-dependence of the $S$-factors of this reaction and of previously measured $(\mathrm{p}, \gamma)$ and $(\mathrm{p}, \mathrm{n})$ reactions on Se and $\mathrm{Sr}$ isotopes can be better reproduced when increasing the JLM imaginary depth by $70 \%$. This can be attributed to a stronger isovector component in the imaginary part. For further details, see [29].

Regarding optical potentials for $\alpha$ particles, see [30] for an extended discussion. There are no microscopic potentials available and attempts to derive a global potential are restricted to fits of Woods-Saxon shapes. Interestingly, despite of the higher Coulomb barrier, $\alpha$ captures at low energies can still be described within a factor of two by the simple potential of [31] which was derived from $\alpha$ scattering at energies of several tens of $\mathrm{MeV}$. However, a trend is found in measured values which often are well predicted theoretically closely above the Coulomb barrier height and exhibiting a slowly widening difference at sub-barrier energies (see, e.g., [32, 33]). This indicates that the barrier transmission may not be described correctly and that an additional dependence on 
barrier height may have to be introduced in the potentials. Another problem may arise due to the formation of $\alpha$ clusters in the compound nucleus. The difficulty in describing low-energy $\alpha$-induced reactions is similar to the well-known problem of predicting halflives of spontaneous $\alpha$-emitters.

There is one remarkable case, ${ }^{144} \mathrm{Sm}(\alpha, \gamma)$, showing the by far strongest discrepancy between experiment and theory at the lowest measured energy of $10.2 \mathrm{MeV}$ [34]. Because of the importance of the extrapolation to $9 \mathrm{MeV}$ for $\gamma$-process nucleosynthesis, it is urgently required that this reaction be remeasured at sub-Coulomb energies!

In general, when performing measurements aiming at an improvement in optical potentials one has to select reactions properly. The potential of interest should give rise to the smallest width of the involved channels because otherwise the sensitivity of the cross section to a variation of the potential is small [29, 35]. For $\alpha$ potentials this can be achieved by studying $(\alpha, \gamma)$ around the Coulomb barrier or in $(\alpha, n)$ and $(\mathrm{n}, \alpha)$ reactions. For the proton potential, $(\mathrm{p}, \mathrm{n})$ or $(\mathrm{n}, \mathrm{p})$ reactions can be useful. This has also to be considered when fitting optical potentials to reaction data. Only reactions sensitive to the optical potential will provide useful constraints. Such fits including reaction data may be of particular importance because it has been pointed out recently that there may be a fundamental difference between potentials derived from scattering on a "cool" target and emission from a "hot" nucleus [36]. A dependence on nuclear temperature may have to be included in the potentials.

\section{Direct capture}

\subsection{Importance for neutron-rich nuclei}

The vast majority of reactions relevant for astrophysics can be treated in the statistical model. However, the nuclear level density is low in nuclei which are light $(A \lesssim 20)$ or have closed shells. Towards the driplines, the neutron- or proton-separation energies are decreasing which leads to formation of the compound nucleus at low excitation energy and thus with a low level density at that formation energy. In all these cases, the Hauser-Feshbach model will not be applicable anymore and has to be supplemented by accounting for resonant and direct processes. This is straightforward provided the required nuclear properties are known and the mechanisms are clearly separated. The latter will be the case when one of the reaction mechanisms dominates. If two of the mechanisms exhibit similar strength, optical potentials may have to be modified to account for the increased flux into the other reaction channel. Additionally, coherent summation of the channels may be required to allow for interference effects.

Even in heavy, closed shell nuclei direct capture may have a non-negligible contribution as, e.g., was demonstrated in [37] where a discrepancy between an activation and a resonance measurement of ${ }^{208} \mathrm{~Pb}(\mathrm{n}, \gamma)$ was shown to be caused by this mechanism. It becomes dominant in the absence of resonances. More specifically, it will be nonnegligible for targets close to the driplines. Such nuclides are produced in the rp-process 


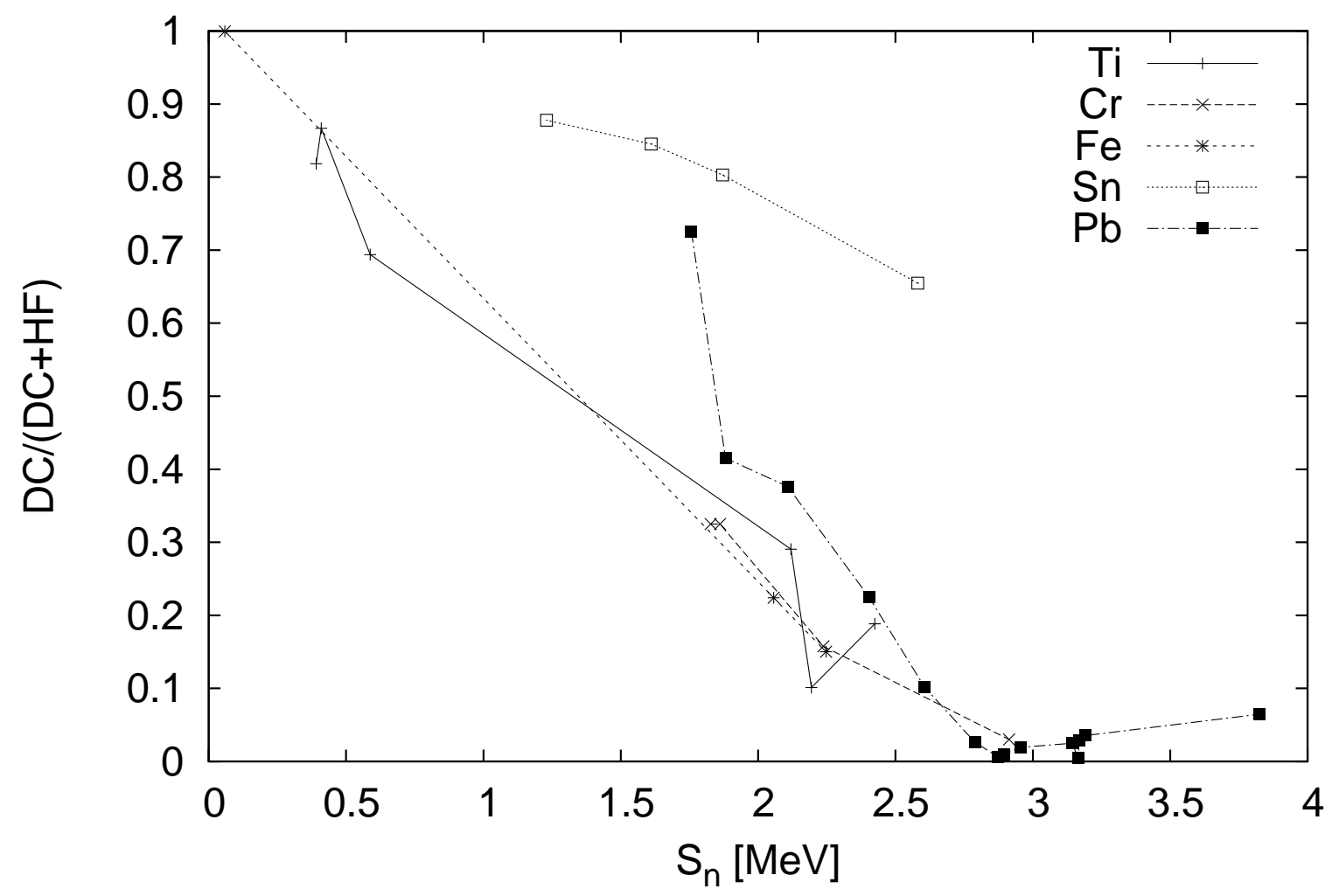

Figure 1. Relation between direct neutron capture and compound capture as function of neutron separation energy for isotopes of Ti, Cr, Fe [38, Sn [39], and $\mathrm{Pb}$ 37.

on the proton-rich side and the r-process at the neutron-rich side of the nuclear chart. Figure 1 shows the increasing importance of direct neutron capture with decreasing neutron separation energies. (It is to be noted that these estimates just compare compound and direct cross section without accounting for interference effects.)

\subsection{Statistical direct capture}

It has been shown [37] that the direct cross section is very sensitive to the predicted properties of the final states and that different microscopic models yield vastly different results. The sensitivity is much higher than in the case of compound reactions because no averaged quantities are used. In order to circumvent the problem of the exact prediction of states it has been suggested [40, 41, 42] to employ averaged properties also for direct captures, i.e. to replace the sum over discrete final states by an integration over a level density. Determination of the nuclear level density poses the same problem as in the case of compound reactions. Additionally, spectroscopic factors have to be predicted. Previously, these were assumed to be constant [42] or energy-dependent [40, 41, 43].

The spectroscopic factors describe the overlap between the antisymmetrized wave functions of target + nucleon and the final state. The number of final state configurations 


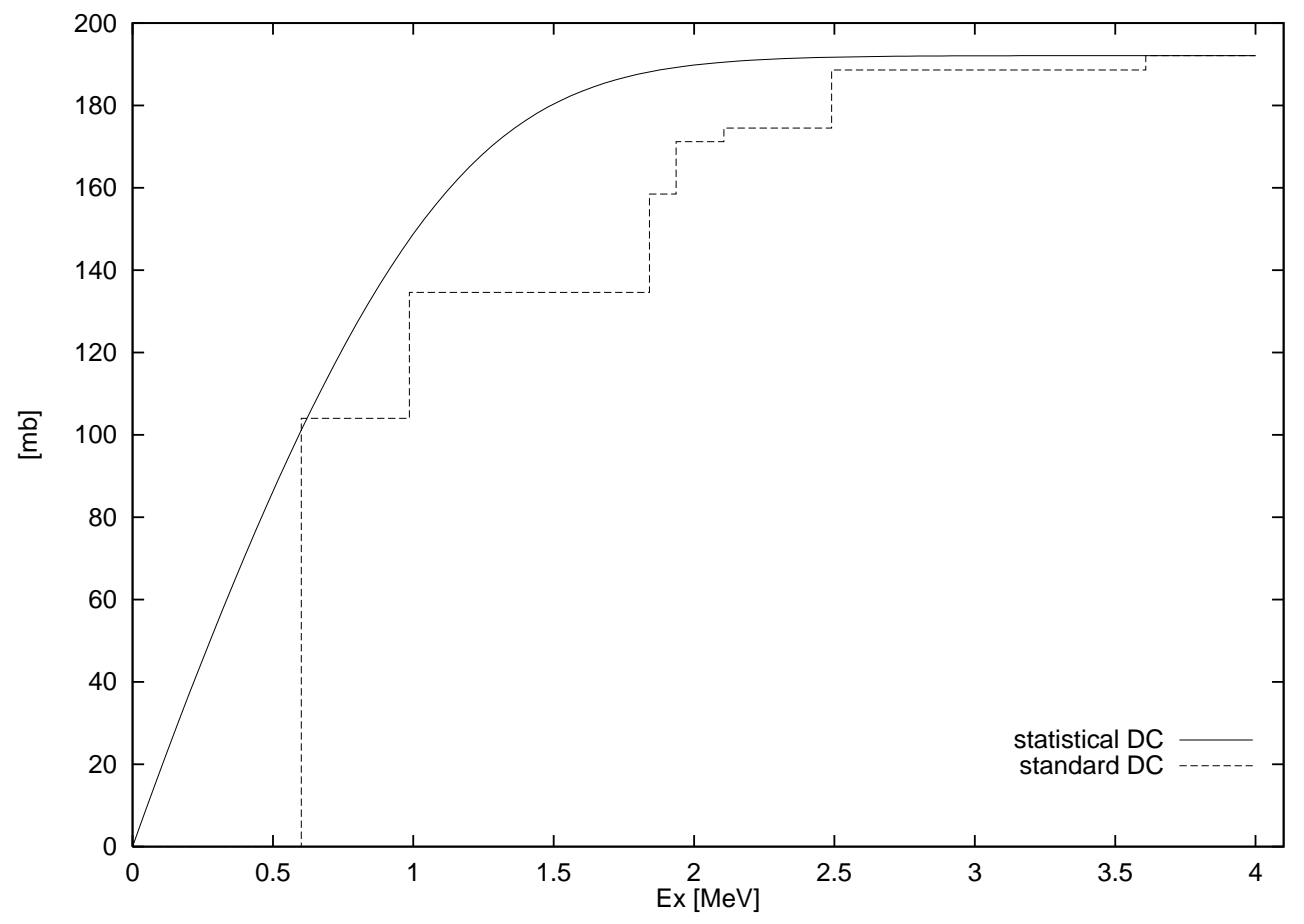

Figure 2. Sums of the contributions to the total direct capture cross sections with the averaged (statistical) DC model and the standard DC for ${ }^{136} \mathrm{Xe}(\mathrm{n}, \gamma)$ as function of excitation energy 41]

increases with increasing excitation energy and the overlap of initial and final state wavefunctions decreases. Thus, also the spectroscopic factor $S$ decreases. In a simple approach, the energy dependence of the spectroscopic factor can be parameterized by a Fermi function with

$$
S=\frac{1}{1+e^{\frac{E_{\mathrm{x}}-E^{*}}{\Delta}}}
$$

and the parameters $E^{*}, \Delta$. Despite of its simplicity, direct capture cross sections can be well described by this approach [41]. Figure 2 shows a comparison between averaged direct neutron capture and standard neutron capture calculated in the Lane-Lynn model [44] for ${ }^{136} \mathrm{Xe}$ and using the spectroscopic factors shown in Figure 3. For one-nucleon capture, $S$ can also be derived from the occupation probability $v^{2}$ for the target state into which the nucleon is captured. It can be calculated, e.g., by employing BCS [4] or Lipkin-Nogami pairing [38, 37]. For the case of one-nucleon capture on even-even nuclei, the spectroscopic factor $S$ for capture can be reduced to $S=1-v^{2}$ [38, 45].

Recently, it was found that thermal excitation of target nuclei reduces the sensitivity to the parity dependence in the nuclear level density [4]. We can expect a similar effect for direct capture, although the number of possible transitions is more limited [4]. A detailed discussion will be presented in a forthcoming paper [46], along with large-scale calculations in the averaged direct capture model with excitation-energy dependent level 


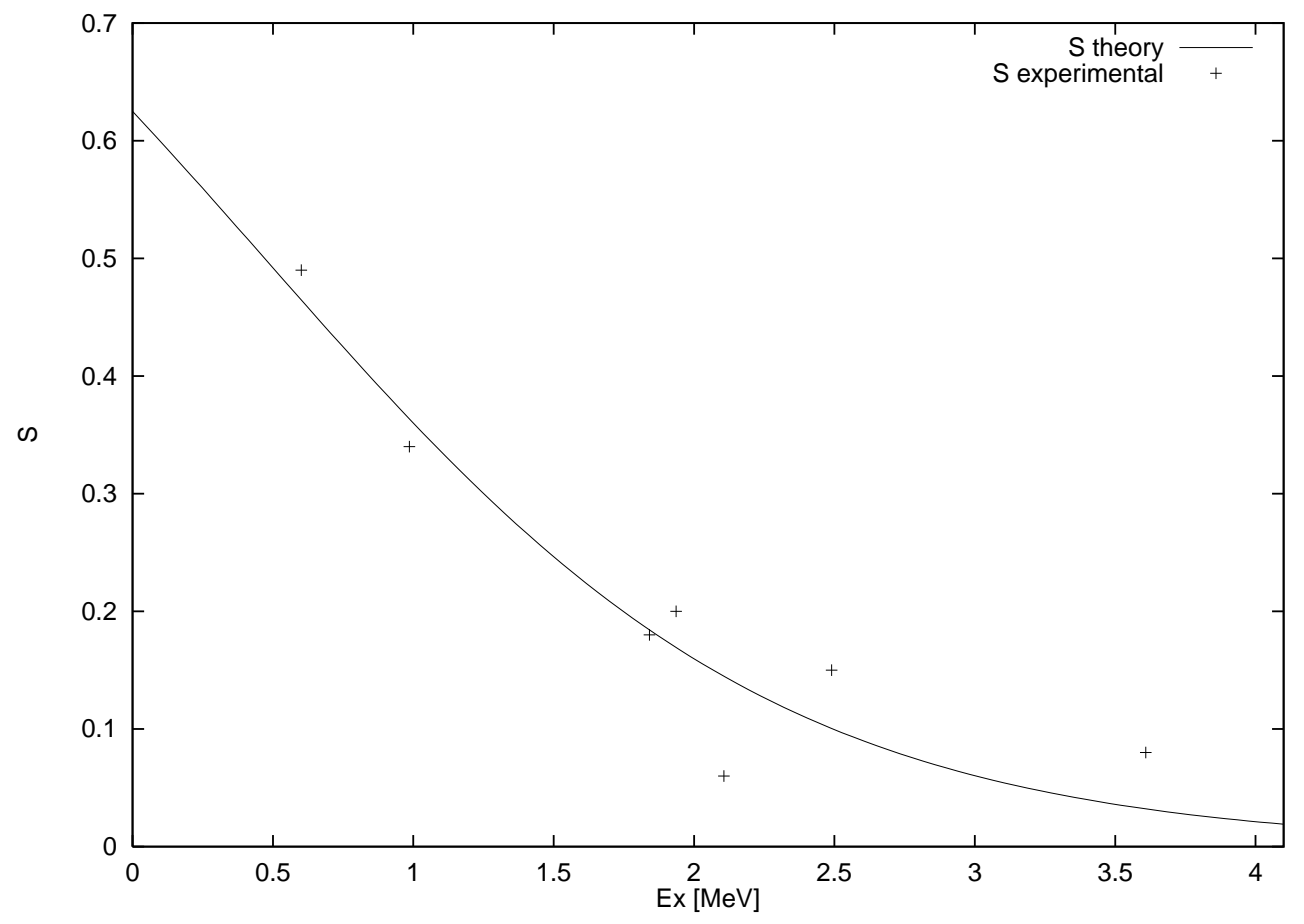

Figure 3. Comparison between experimental and averaged (equation 1) spectroscopic factors as function of excitation energy for ${ }^{136} \mathrm{Xe}+\mathrm{n}$ [4].

densities and spectroscopic factors.

\section{Summary and conclusion}

The sensitivities of reaction cross sections to predicted nuclear properties were discussed here for compound and direct reactions. Obviously, strong single resonances in the relevant energy range will also have considerable impact on the resulting astrophysical reaction rates. Because the resonance energies have to be predicted to a few hundred $\mathrm{keV}$, this poses a continuous challenge to nuclear theory. For a detailed study of the actual impact of rate variations on nucleosynthesis in massive stars for nuclides with $28 \leq A \leq 80$, see [47].

\section{References}

[1] Descouvemont P and Rauscher T 2006 Nucl. Phys. A777 137

[2] Rauscher T, Kratz K-L and Thielemann F-K 1997 Phys. Rev. C 551613

[3] Hauser W and Feshbach H 1952 Phys. Rev. 87366

[4] Mocelj D, Rauscher T, Martínez-Pinedo G, Langanke K, Pacearescu L, Faessler A, Thielemann F-K, and Alhassid Y 2007 Phys. Rev. C 75045805

[5] Rauscher T and Thielemann F-K 2000 At. Data Nucl. Data Tables 751

[6] Rauscher T and Thielemann F-K 2001 At. Data Nucl. Data Tables 7947 
[7] Rauscher T and Thielemann F-K 1998 Stellar Evolution, Stellar Explosions, and Galactic Chemical Evolution ed A Mezzacappa (Bristol: IOP) p 519

[8] Goriely S 1998 Phys. Lett. B 43610

[9] Rauscher T, code NON-SMOKER ${ }^{\mathrm{WEB}}$, unpublished (http://nucastro.org/nonsmoker.html)

[10] Goriely S and Khan E 2002 Nucl. Phys. A706 217

[11] Tsoneva N, Lenske H and Stoyanov C 2004 Phys. Lett. B 586 213; Tsoneva N et al 2008 J. Phys. G 35014047

[12] Vretenar D, Paar N, Ring P, and Lalazissis G A 2002 Nucl. Phys. A692 496

[13] Paar N, Nikšić T, Vretenar D, and Ring P 2005 Phys. Lett. B 606288

[14] Vretenar D 2008 J. Phys. G 35014039

[15] Piekarewicz J 2006 Phys. Rev. C 73044325

[16] Liang J, Cao L-G and Ma Z-Y 2007 Phys. Rev. C 75054320

[17] Adrich P et al 2005 Phys. Rev. Lett. 95132501

[18] Klimkiewicz A et al 2007 Nucl. Phys. A788 145

[19] Boretzky K et al, this volume

[20] Zilges A, Volz S, Babilon M, Hartmann T, Mohr P, and Vogt K 2002 Phys. Lett. B 54243

[21] Rauscher T, Thielemann F-K, Görres J, and Wiescher M 2000 Nucl. Phys. A675 695

[22] Woosley S E, Fowler W A, Holmes J A, and Zimmerman B A 1978 At. Data Nucl. Data Tables 22371

[23] Mohr P et al 1997 Phys. Rev. C 551523

[24] Jeukenne J P, Lejeune A and Mahaux C 1977 Phys. Rev. C 1680

[25] Lejeune A 1980 Phys. Rev. C 211107

[26] Bauge E, Delaroche J P and Girod M 1998 Phys. Rev. C 581118

[27] Bauge E, Delaroche J P and Girod M 2001 Phys. Rev. C 63024607

[28] Gyürky Gy et al 2003 Phys. Rev. C 68055803

[29] Kiss G G, Gyürky Gy, Elekes Z, Fülöp Zs, Somorjai E, Rauscher T, and Wiescher M 2007 Phys. Rev. C 76 055807; Kiss G G et al 2008 J. Phys. G 35014032

[30] Demetriou P, this volume

[31] McFadden L and Satchler G R 1966 Nucl. Phys. 84177

[32] Özkan N et al 2007 Phys. Rev. C 75025801

[33] Basunia M S, Shugart H A, Smith A R, and Norman E B 2007 Phys. Rev. C 75015802

[34] Somorjai E et al 1998 Astron. Astrophys. 3331112

[35] Gyürky Gy et al 2006 Phys. Rev. C 74025805

[36] Avrigeanu M, von Oertzen W and Avrigeanu V 2006 Nucl. Phys. A764 246

[37] Rauscher T, Bieber R, Oberhummer H, Kratz K-L, Dobaczewski J, Möller P, Sharma M M 1998 Phys. Rev. C 572031

[38] Rauscher T, Böhmer W, Kratz K-L, Balogh W, Oberhummer H 1995 Proc. Int. Conf. Exot. Nuclei and Atomic Masses ed. M de St Simon, O Sorlin (Gif-sur-Yvette: Éditions Frontières) p 683

[39] Balogh W, Bieber R, Oberhummer H, Rauscher T, Kratz K-L, Mohr P, Staudt G 1994 Proc. Europ. Workshop Heavy Element Nucleosynthesis, Budapest, ed E Somorjai, Zs Fülöp (Debrecen: ATOMKI) p 67; http://arxiv.org/abs/nucl-th/9404010

[40] T. Rauscher, report IKP1996-02017, TU Wien (1996).

[41] E. Holzer, Statistische Berechnung von direktem Neutroneneinfang, Master thesis, TU Wien (1997).

[42] S. Goriely, Astron. Astrophys. 325, 414 (1997).

[43] R. Ejnisman, I.D. Goldman, K.S. Krane, P. Mohr, Y. Nakazawa, E.B. Norman, T. Rauscher, and J. Reel, Phys. Rev. C 58, 2531 (1998).

[44] Lane A M and Lynn J E 1960 Nucl. Phys. 17 563; Nucl. Phys. 17586

[45] Glendenning N K 1983 Direct Nuclear Reactions (New York: Academic Press)

[46] Rauscher $\mathrm{T}$ et al 2008, in preparation

[47] Hoffman R D, Woosley S E, Weaver T A, Rauscher T, and Thielemann F-K 1999 Astrophys. J. $\mathbf{5 2 1} 735$ 